\title{
EARTHQUAKES AND TESSELLATIONS OF TEICHMÜLLER SPACE
}

\author{
BY
}

PETER WATERMAN AND SCOTT WOLPERT ${ }^{1}$

\begin{abstract}
A brief exposition of the Teichmüller and earthquake theory is given for the once punctured torus. Using these ideas. computer plots. of the tessellation of Teichmüller space by the mapping class group and of the earthquake paths leaving a point. were generated. The algorithms for generating the plots are discussed.
\end{abstract}

I. The deformation space, the Teichmüller space $T$, of a Riemann surface $R$ of finite hyperbolic area is a complex manifold. The mapping class group $\mathfrak{N}$ of $R$ acts as a group of biholomorphic self-maps of $T$. Several $M$ invariant geometries for $T$ have been introduced.

A natural example is provided by Thurston's construction of special 1-parameter families of deformations-earthquakes. For the classical case, $\mathcal{R}$ the once punctured torus, we shall sketch the fundamentals of Teichmüller and earthquake theory. Particular attention will be paid to the action of the mapping class group and to certain special nonholomorphic coordinates for $\pi$. Using this information computer plots of the tessellation of $\div$ by $: T h$ are generated in two different coordinate systems. The earthquake paths in Teichmüller space emanating from a point are graphed for several distinct points.

There are many open questions concerning earthquakes and the action of the mapping class group. We hope these pictures will provide evidence for further results.

The following paragraphs are expository in nature-references for this material are $[1,2,3,5]$.

II. We wish to give a brief description of the Teichmüller theory of a once punctured torus, $\Omega$. By the above, it suffices to consider the totality of hyperbolic geometries for $\bar{s}$.

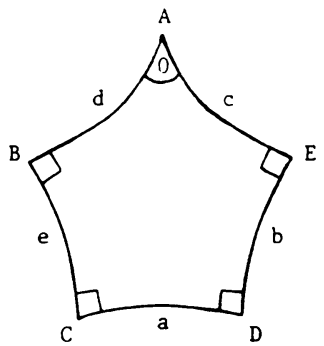

Received by the editors February 9, 1982.

1980 Mathematics Subject Classification. Primary 30F35, 30F10, 53A35.

'Partially supported by National Science Foundation Grant \# MCS78-03838; Alfred P. Sloan Fellow 1981-1983. 
A complete finite area hyperbolic geometry for a torus with one point removed is obtained from an elementary construction. Consider the pentagon $A B C D E$ in the hyperbolic plane. The vertex angle at $A$ is zero and the remaining angles are right angles. We further require that the sides $b$ and $e$ are of equal length: $l / 2$. From trigonometry the pentagon is uniquely determined by the quantity $l$ which may be prescribed arbitrarily. Now identify in pairs the sides $a \sim a^{\prime}, c \sim c^{\prime}$ and $d \sim d^{\prime}$ of two identical pentagons $A B C D E$ and $A^{\prime} B^{\prime} C^{\prime} D^{\prime} E^{\prime}$. The resulting geometric object is a cylinder $C$ with one cusp. The boundaries of $C$ are isometric geodesics of length $l$ and may be identified to form an oriented hyperbolic surface $\delta$ : a once punctured torus. We now wish to count the degrees of freedom for the construction. First, there is the length $l$ of a boundary of $C$. Second, we observe that there is a single degree of freedom for the last identification. On each boundary of $C$ there is an endpoint of the image of $a$. In identifying the boundaries of $e$, we may arbitrarily prescribe the displacement between the images of these points. Perhaps a word of clarification is in order. The above construction also provides a natural basis for the fundamental group of $\delta: \pi_{1}(\delta)$. The base point is the image of the vertex $C$. The first generator, $\alpha$, is the image in $\mathcal{S}$, of the arc $e \cup e^{\prime}$ traversed in the positive sense. Let a real number $\tau$ now be prescribed. The second generator $\beta$ is a union of two arcs: commence at $C$, traverse $e \cup e^{\prime}$ in the positive direction for a distance $\tau$, then proceed to the left along the image of $a$ in $\delta$ to the image of $C$. The key point is that the identification of $e \cup e^{\prime}$ and $b \cup b^{\prime}$ is made so that $D$ will be $\tau$ units from $C$ along the identification locus. Hence, the above rule prescribes a closed curve. In conclusion, the quantities $\tau \in \mathbf{R}, l \in \mathbf{R}^{+}$are the parameters for the construction: every hyperbolic geometry for the once punctured torus is obtained in this way. The pair $(\tau, l)$ are the Fenchel-Nielsen coordinates for the Teichmüller space, $\mathcal{T}$.

We now consider the question of uniqueness. The fundamental group, $\pi_{1}(\delta)$, can be generated by two elements. Partition the set of generating pairs by the orbits of the inner automorphism group $\operatorname{Inn}\left(\pi_{1}(\delta)\right)$. To a hyperbolic geometry and equivalence class of generators of $\pi_{1}(\delta)$ is associated biuniquely a pair $(\tau, l)$. Consequently, for a fixed hyperbolic geometry there is a distinct pair $(\tau, l)$ for each equivalence class of generators. The different equivalence classes are related by the outer automorphism group of $\pi_{1}(\delta)$; the extended mapping class group $\tilde{\mathscr{N}}$ which therefore acts on $\mathcal{T}$. It is known that $\tilde{\mathscr{T}}$ has an index 2 orientation preserving subgroup $\Re$ isomorphic to $\operatorname{SL}(2, \mathbf{Z})$ and that the action of $\tilde{\mathscr{N}}$ is properly discontinuous. We shall describe the action in various coordinate systems. In [7] it is shown that in the Fenchel-Nielsen coordinates the 2 -form $d \tau \wedge d l$ is $\Re$ invariant and hence that the action of $\pi$ is euclidean-area preserving.

Before describing other coordinates we wish to mention the earthquake deformations introduced by Thurston. An earthquake is a 1-parameter deformation and thus corresponds to a curve in Teichmüller space. The prototype is for a simple closed geodesic. If, for example, we choose the geodesic $\alpha$ from the above discussion the deformation is the horizontal curve, $l=$ constant in $(\tau, l)$ coordinates. Specifically, to earthquake an amount $t$ on $\alpha$, the surface $\delta$ is cut open along $\alpha$, one side is rotated by an amount $t$ (a natural orientation exists) and finally the sides are identified in 
their new position. Thurston observed that the simple closed geodesics may be replaced by measured geodesic laminations and that the construction can be generalized to this context. Perhaps the following theorem of Thurston suggests the central role of earthquakes to deformation theory.

THEOREM (THURSTON [5]). Let $\widetilde{T}_{g, n}$ be the Teichmüller space of a Riemann surface of genus $g$ with $n$ punctures $2 g-2+n>0$. Given $p, q \in \mathcal{T}_{g, n}$, there exists a unique left earthquake connecting $p$ to $q$.

An open question is to find a differential equation for which the earthquakes are the trajectories. Preliminary results in this direction are obtained in [7].

Some notation is required before we consider other coordinate systems. Given $\gamma \in \pi_{1}(\delta)$, let $l(\gamma)$ be the length of the unique geodesic freely homotopic to $\gamma$. Further let $\operatorname{tr} \gamma$ be the common absolute trace of the conjugacy class of $\gamma \in \pi_{1}(\delta)$ for $\pi_{1}(\S)$ the Fuchsian group uniformizing $\delta$. The identity

$$
\operatorname{tr} \gamma=2 \cosh l(\gamma) / 2
$$

is elementary. For $\alpha, \beta$, the generators of $\pi_{1}(\S)$, set $x=\operatorname{tr} \alpha, y=\operatorname{tr} \beta, z=\operatorname{tr} \alpha \beta$. The quantities $x, y, z$ satisfy $x^{2}+y^{2}+z^{2}=x y z ; x, y, z>2$. We have [2,3].

THEOREM. The Teichmüller space $\mathcal{T}$ of the once punctured torus is the sublocus of $x^{2}+y^{2}+z^{2}=x y z$ satisfying $x, y, z>2$.

The approach to describing the change of coordinates from $(\tau, l)$ to $(x, y, z)$ is based on the following:

LEMMA (GREENBERG). Let $\delta$ be a once-punctured torus and $\gamma$ a free homotopy class intersecting $\alpha$ once. Then

$$
\frac{d^{2}}{d \tau^{2}} \operatorname{tr} \gamma=\frac{1}{4} \operatorname{tr} \gamma
$$

Proof. The uniformization group $\pi_{1}(\delta)$ is obtained from a free group by an HNN extension [6]. In this representation we assume that $\alpha$ lifts to the imaginary axis in the upper half-plane, then $\gamma$ corresponds to a matrix product

$$
F\left(\begin{array}{ll}
e^{\tau / 2} & 0 \\
0 & e^{-\tau / 2}
\end{array}\right) G
$$

where $F, G$ are constant and $\tau$ is the Fenchel-Nielsen parameter. Trivially, we have

$$
\frac{d^{2}}{d \tau^{2}} \operatorname{tr} F\left(\begin{array}{ll}
e^{\tau / 2} & 0 \\
0 & e^{-\tau / 2}
\end{array}\right) G=\frac{1}{4} \operatorname{tr} F\left(\begin{array}{ll}
e^{\tau / 2} & 0 \\
0 & e^{-\tau / 2}
\end{array}\right) G
$$

The problem in calculating the change of variables is to express $l(\beta)$ in terms of $\tau$ and $l=l(\alpha)$. A simple argument shows that $\operatorname{tr} \beta$ achieves its minimum when $\tau=0$. Hence $(d / d \tau) \operatorname{tr} \beta$ vanishes when $\tau=0$ and $\operatorname{tr} \beta=c_{0} \cosh \tau / 2$ where $c_{0}$ is the value 
of $\operatorname{tr} \beta$ for the special position $\tau=0$. In this case the edge $a$ of the original pentagon $A B C D E$ becomes the geodesic $\beta$ and an elementary exercise in hyperbolic trigonometry shows that $\cosh l(a) / 2=\operatorname{coth} l(\alpha) / 2$. Substitution yields

$$
\cosh l(\beta) / 2=\operatorname{coth} l / 2 \cosh \tau / 2
$$

or, using $x, y$,

$$
x=2 \cosh l / 2, \quad y=2 \operatorname{coth} l / 2 \cosh \tau / 2 .
$$

We note that the 2-form $(4 d x \wedge d y) /(x y-2 z)$ is the image of the FenchelNielsen area form $d \tau \wedge d l$.

Setting $a=x /(y z), b=y /(x z), c=z /(x y)$ the Teichmüller space is the simplex $a+b+c=1, a, b, c>0$. For reference the invariant 2 -form is $(d a \wedge d b) /(a b c)$. We shall describe the action of the modular group in these coordinates. It does not act effectively: the quotient by the kernel of the action is isomorphic to $\operatorname{PSL}(2, \mathbf{Z})[8]$. It is most convenient to consider a reflection group $P \tilde{\Gamma}(2)$ with index 2 subgroup $P \Gamma(2)=\left\{\left(\begin{array}{ll}a & b \\ c & d\end{array}\right) \in \operatorname{PSL}(2, \mathbf{Z}) \mid\left(\begin{array}{ll}a & b \\ c & d\end{array}\right) \equiv\left(\begin{array}{ll}1 & 0 \\ 0 & 1\end{array}\right) \bmod 2\right\}$. Its generators $Q, R, S$ act as follows:

$$
\begin{gathered}
Q(a, b, c)=\left(1-a, \frac{a b}{1-a}, \frac{a c}{1-a}\right), \quad S(a, b, c)=\left(\frac{c a}{1-c}, \frac{c b}{1-c}, 1-c\right), \\
R(a, b, c)=\left(\frac{b a}{1-b}, 1-b, \frac{b c}{1-b}\right)
\end{gathered}
$$

and each leave $(d a \wedge d b) /(a b c)$ invariant (modulo sign).

Finally, the fixed point loci of the above involutions bound a fundamental domain $\Delta=\{(a, b, c) \in \mathcal{T} \mid a, b, c \leqslant 1 / 2\}$ for $P \tilde{\Gamma}(2)[8]$.

III. Programming was done in BASIC on an HP-85 minicomputer with plotter.

The first three plots are tessellations of the Teichmüller space $\mathcal{T}$ by $P \tilde{\Gamma}(2)$. In plots (1) and (2), $\sigma$ is represented in the $(a, b, c)$ coordinates. The lines $a=1 / 2, b=$ $1 / 2, c=1 / 2$ bound the fundamental domain $\Delta$. For $V \in P \tilde{\Gamma}(2), V(a=1 / 2)$, $V(b=1 / 2), V(c=1 / 2)$ bound the image domain $V(\Delta)$. For example, to plot $Q R Q(a=1 / 2)$ the transformations $Q, R, Q$ given above are successively applied to a sequence of points on the line $a=1 / 2$. The resulting sequence lies on the curve $Q R Q(a=1 / 2)$ and, providing the spacing is sufficiently small, the result is a visually smooth curve. The elements of $P \tilde{\Gamma}(2)$ are enumerated according to word length. Hence the tessellation begins with $\Delta$ and expands outward. To achieve this consider the presentation,

$$
P \tilde{\Gamma}(2)=\left\langle Q, R, S \mid Q^{2}=R^{2}=S^{2}=1\right\rangle .
$$

Accordingly, reduced words in $Q, R, S$ are in 1-1 correspondence with ternary integers without repeated digits in which a leading zero is permitted, e.g. 012013. A simple rule for generating such numbers is that the possible digits to the left of a given digit are obtained by adding 1 or $2 \bmod 3$ to the given digit. In plot (3) the tessellation is given in the $(\tau, l)$ coordinates. Note that the regions are all of equal Euclidean area for this plot. 


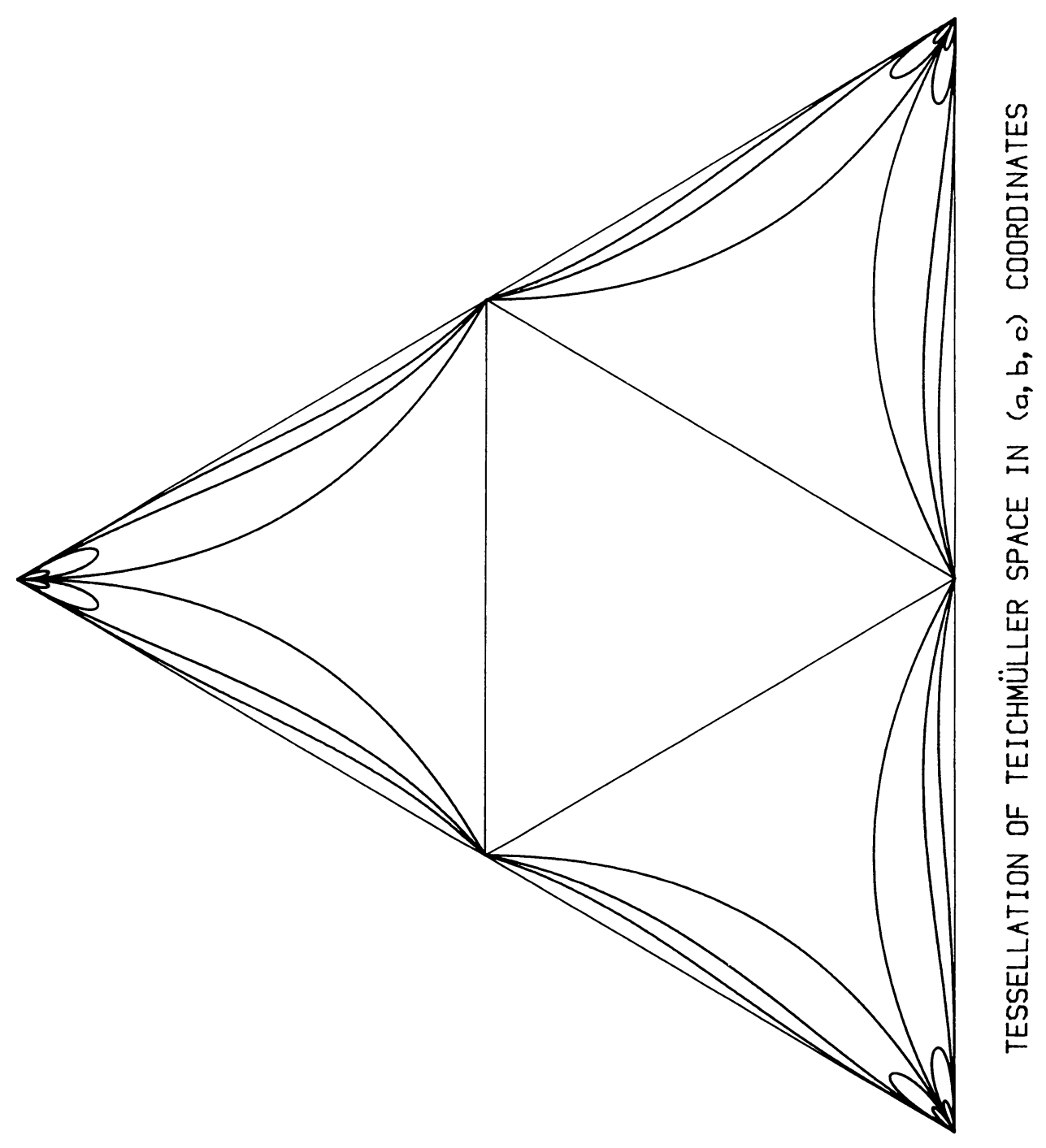




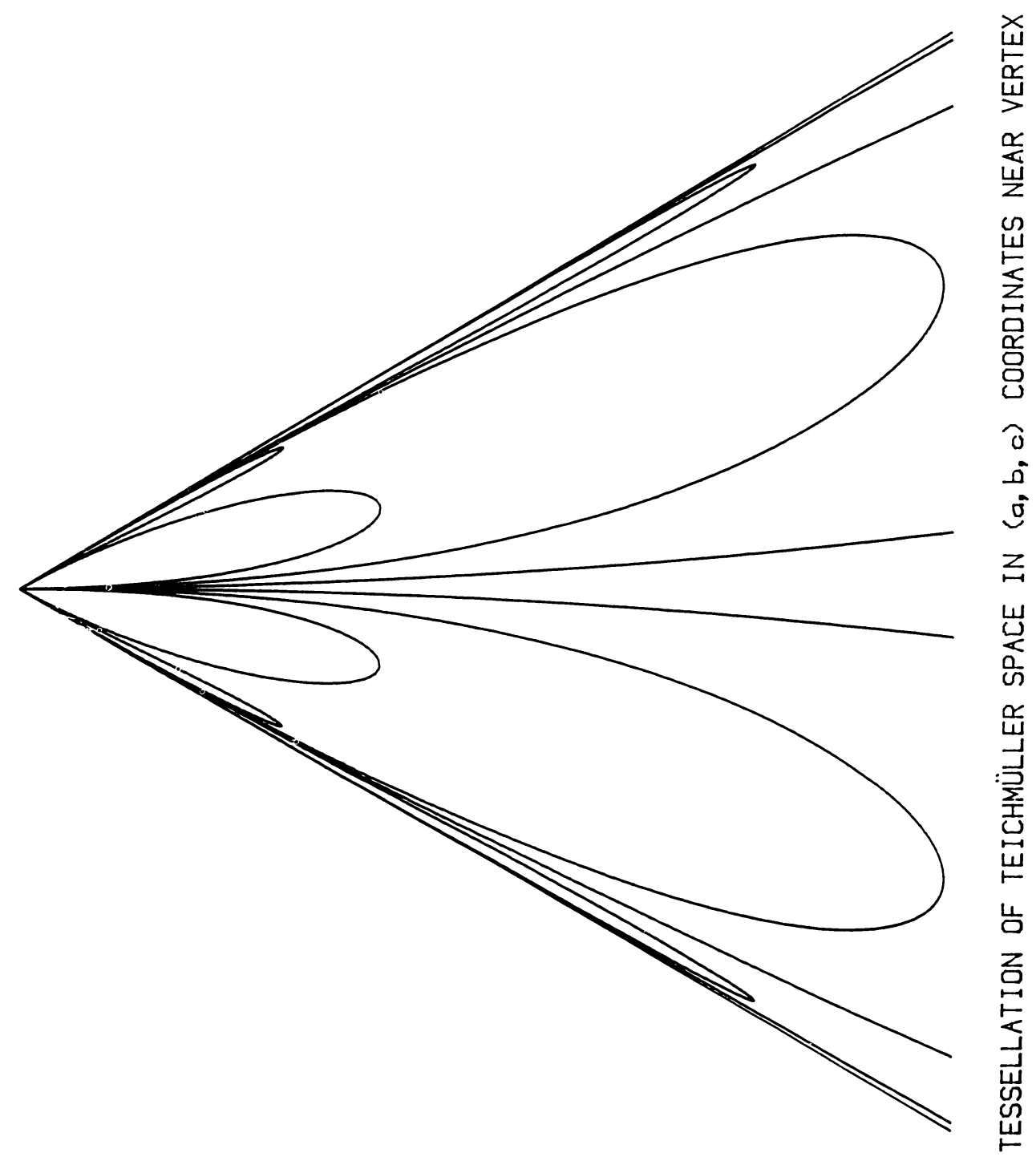


TESSELLATIONS OF TEICHMÜLLER SPACE

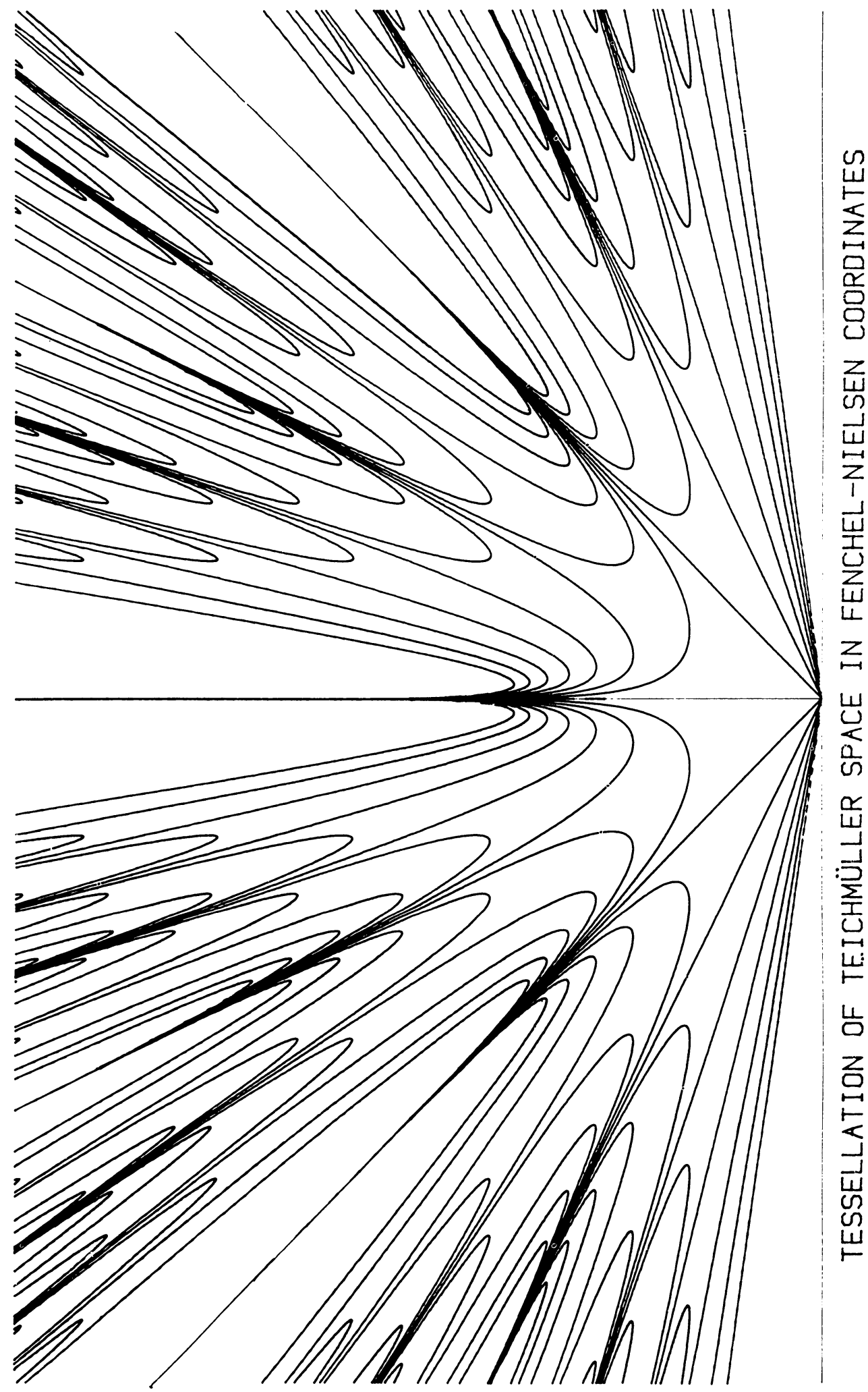




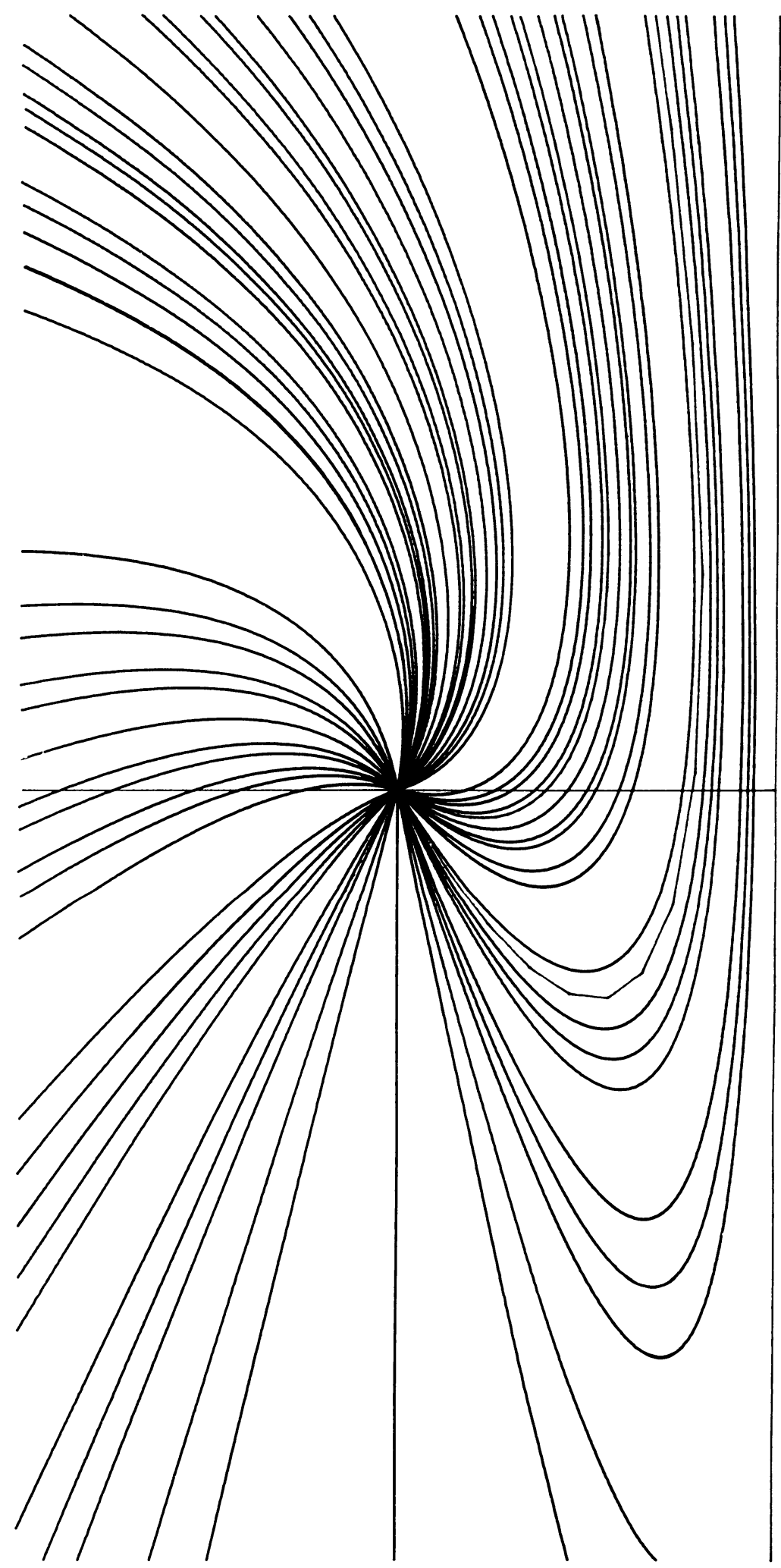

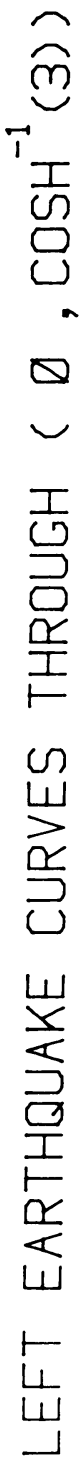




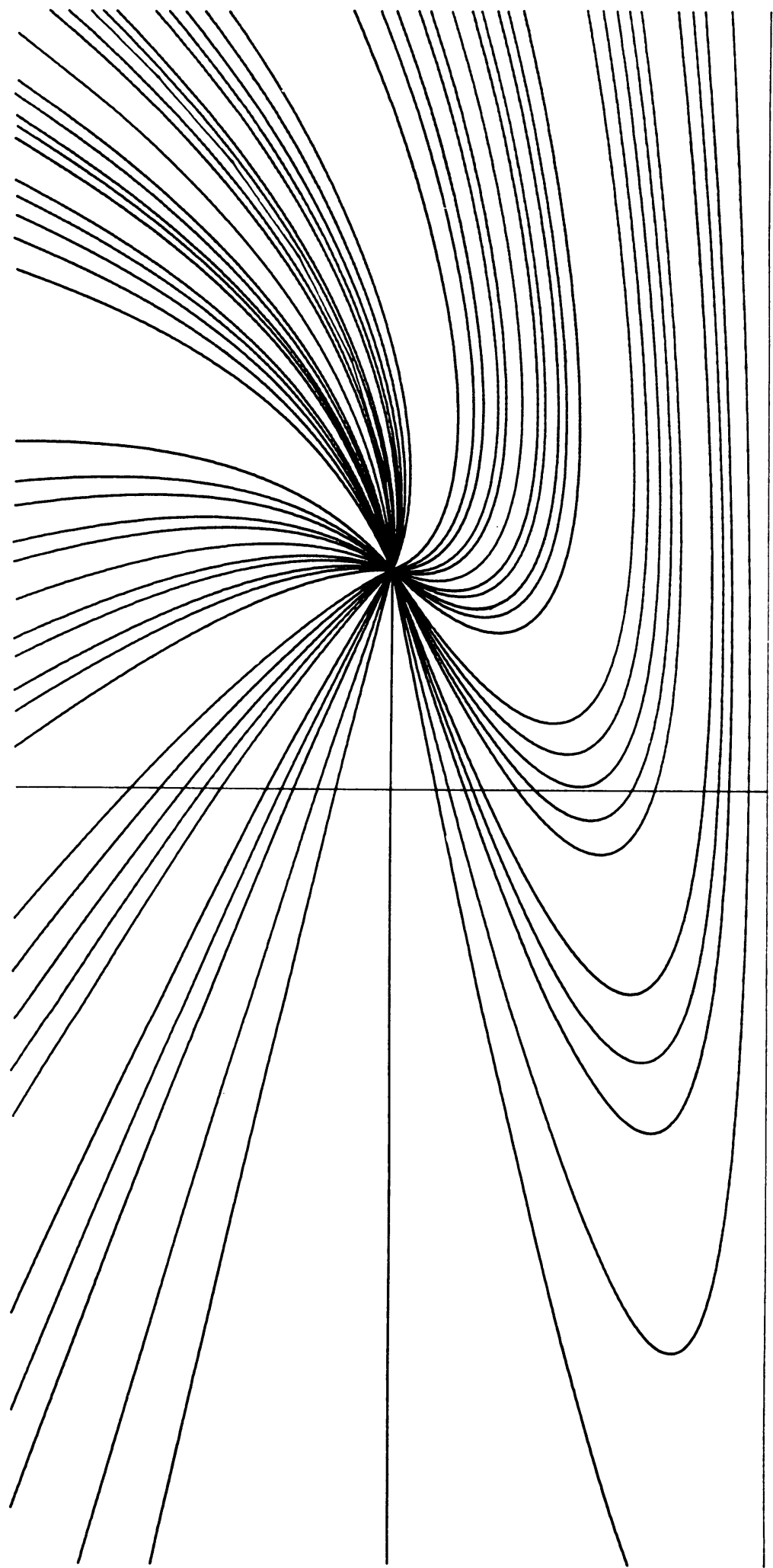

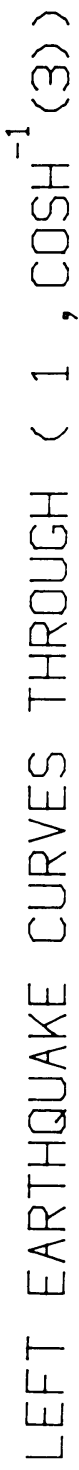




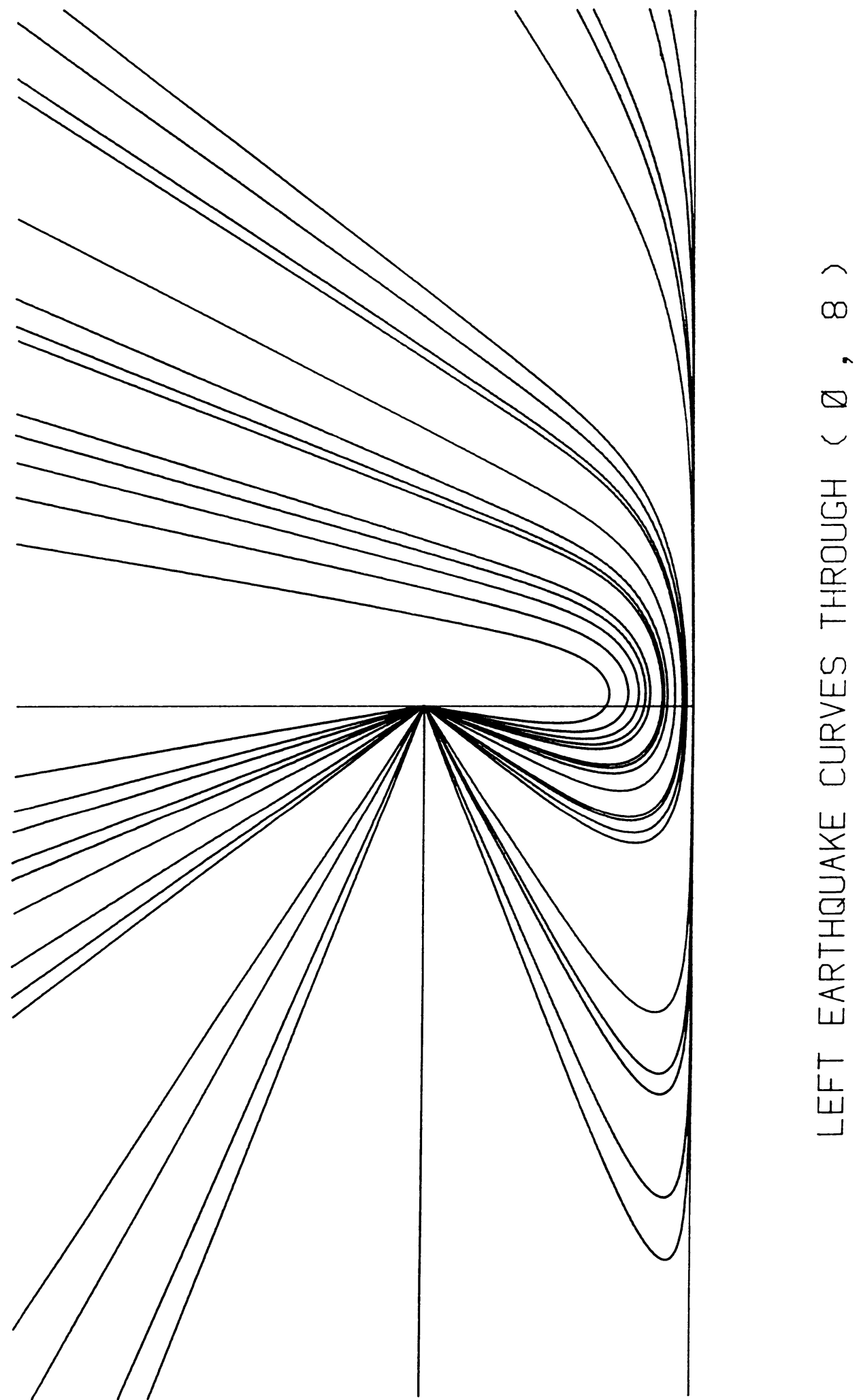


The last three plots are of the left earthquakes leaving a point of Teichmüller space. We have only considered those corresponding to simple closed geodesics. A theorem of Thurston guarantees that such deformations are dense in the space of earthquakes. Our method for computing earthquakes is based on the observation that if $V \in \tilde{\mathscr{N}}$ then $V(l=$ constant $)$ is the earthquake for the simple closed geodesic associated to the conjugacy class of $V^{-1}(\alpha)$ in $\pi_{1}(\delta)$. The plots will be given in the Fenchel-Nielsen coordinates $(\tau, l)$. Specifically, fix a point $p$ in $\sigma$ and let $\left(\tau_{0}, l_{0}\right)$ be the coordinates of $V(p)$ then $V^{-1}\left(\tau \leqslant \tau_{0}, l=l_{0}\right)$ or $V^{-1}\left(\tau \geqslant \tau_{0}, l=l_{0}\right)$ is a left earthquake emanating from $p$ according as $V$ is orientation preserving or reversing. This approach also requires an enumeration of the group $\tilde{\Re}$. In the fourth plot, the point $\left(0, \cosh ^{-1}(3)\right)$ corresponds to the square torus whose Euclidean lattice has generators 1 and $i$.

\section{REFERENCES}

1. W. Abikoff, Topics in the real analytic theory of Teichmüller space, Lecture Notes in Math., vol. 820 , Springer-Verlag, New York, 1980.

2. R. Fricke and F. Klein, Vorlesungen über die Theorie der Automorphen Funktionen, Teubner, Leipzig, 1912; Johnson Reprint, 1965.

3. L. Keen, On fundamental domains and the Teichmüller modular group, Contributions to Analysis, (L. Ahlfors et al., eds.), Academic Press, New York, 1974.

4. S. Kerckhoff, The Nielsen realization problem, preprint.

5. W. Thurston, On the geometry and topology of 3-manifolds, Princeton Univ. Notes.

6. S. Wolpert, The Fenchel-Nielsen deformation, Ann. of Math. 115 (1982), 501-528.

7. On the symplectic geometry of deformations of a hyperbolic surface, preprint.

8. On the Kähler form of the moduli space of once punctured tori, preprint.

Department of Mathematics, University of Maryland, College Park, Maryland 20742 (Current address of Scott Wolpert)

Current address (Peter Waterman): Department of Mathematics, Temple University, Philadelphia, Pennsylvania 19122 